\title{
Almost sure invariance principle for some maps of an interval
}

\author{
KRYSTYNA ZIEMIAN \\ Instytut Matematyki, Uniwersytet Warszawski, PKiN IXp, 00-901 Warszawa, Poland
}

(Received 7 February 1985)

\begin{abstract}
We prove an almost sure invariance principle and a central limit theorem for the process $\left(F \circ f^{n}\right)_{n \geq 0}$, where $f$ is a map of an interval with a non-positive Schwarzian derivative whose trajectories of critical points stay far from the critical points, and $F$ is a measurable function with bounded $p$-variation $(p \geq 1)$.

The almost sure invariance principle implies the Log-log laws, integral tests and a distributional type of invariance principle for the process $\left(F \circ f^{n}\right)_{n \geq 0}$.
\end{abstract}

\section{Introduction}

Let $f$ be a map of an interval $I$ and $F$ a measurable function on $I$. Under some special assumptions (like expansiveness) on $f$, several authors have proved the existence of a probabilistic measure $\mu$ on $I, f$-invariant and absolutely continuous with respect to the Lebesgue measure.

In $1979 \mathrm{~S}$. Wong [9] considered the process $\left(F \circ f^{n}\right)_{n \geq 0}$ and proved a central limit theorem for $f$ piecewise $C^{2}$ and expanding in the case of a weak mixing $\mu$. His method can be used to obtain an analogous theorem for maps of an interval which we consider below.

In $1980 \mathrm{G}$. Keller [4] proved a central limit theorem for expanding $f$ using the ideas of M.I. Gordin [2].

In $1982 \mathrm{~F}$. Hofbauer and G. Keller [3] proved for expanding maps $f$ not only a central limit theorem but also an almost sure invariance principle, from which follow Log-log laws, integral tests and a distributional type of invariance principle.

In our paper all these theorems are proved for maps of an interval with a non-positive Schwarzian derivative considered by M. Misiurewicz [5], W. Szlenk [8] and B. Szewc [7]. A full list of assumptions and the formulation of the main theorems are given in $\$ 1 . \S 2$ contains basic technical estimations of derivatives. In $\$ 3$ we prove an exponential rate of decreasing of the diameter in the sense of Lebesgue measure of the partition $V_{0}^{n-1} f^{-i} \mathscr{A}$, where $\mathscr{A}$ is the partition into the intervals of monotonicity of $f$.

An exponential rate of decreasing of the diameter of the partition $V_{0}^{n-1} f^{-i} \mathscr{A}$ in the sense of an $f$-invariant probabilistic measure is proved in $\S 4$. $\S 5$ contains a proof of an exponential rate of decreasing of mixing coefficient. The proof of the main results formulated in $\S 1$ is given in $\S 6$. 


\section{Assumptions and main results}

Let $I$ be a closed interval, $A(g) \subset I$ its finite subset containing the ends of $I$, and $g: I \rightarrow I$ a continuous map, monotonic on the connected components of the set $I \backslash A(g)$. We assume that $\left.g\right|_{I \backslash A(g)}$ satisfies the following conditions [5]:

(i) $g$ is of class $C^{3}$;

(ii) $g \neq 0$;

(iii) $S g \leq 0,\left(S g=\left(g^{\prime \prime \prime} / g^{\prime}\right)-\frac{3}{2}\left(g^{\prime \prime} / g^{\prime}\right)^{2}\right)$;

(iv) if $g^{p}(x)=x$, then $\left|\left(g^{p}\right)^{\prime}(x)\right|>1$;

(v) there exists a neighbourhood $U$ of the set $A(g)$ such that for all $a \in A, n \geq 0$, $g^{n}(a) \in A(g)$ or $g^{m}(a) \notin U$ for all $m \geq n$;

(vi) for all $a \in A(g)$ there exists a neighbourhood $U_{a}$ of $a$ and constants $\alpha, \omega$, $\delta>0, u \geq 0$ such that

(a) $\alpha|x-a|^{u} \leq\left|f^{\prime}(x)\right| \leq \omega|x-a|^{u}$,

(b) $\left|f^{\prime \prime}(x)\right| \leq \delta|x-a|^{u-1}$

for all $x \in U_{a}$.

$[5$, th. 6.2$]$ ensures the existence of a probabilistic measure $\mu, g^{k}$-invariant for some $k \geq 1$, absolutely continuous with respect to the Lebesgue measure $\lambda$ and such that the system $\left(g^{k}, \mu\right)$ is exact. The map $g^{k}$ also satisfies the conditions (i)-(vi), where $A\left(g^{k}\right)=\bigcup_{i=0}^{k-1} g^{-i}(A(g))$ plays the role of $A(g)$. In the following we will write $f$ instead of $g^{k}$ and $A$ instead of $A\left(g^{k}\right)$.

Let $F:[0,1] \rightarrow \mathbb{R}$ be a function with bounded $p$-variation $p \geq 1$, i.e. $V^{p} F=$ $\sup \sum_{i=1}^{n}\left|F\left(c_{i}\right)-F\left(c_{i-1}\right)\right|^{p}<+\infty$, where the supremum is taken over all finite subsets $\left\{c_{0}, \ldots, c_{n}\right\}, c_{0}<c_{1}<\cdots<c_{n}$, of the interval $I$. We assume that $\int_{I} F d \mu=0$.

We will treat $\left(F \circ f^{n}\right)_{n \geq 0}$ as a stochastic process on the probabilistic space $([0,1], \mathscr{B}, \mu)(\mathscr{B}$ is the Borel $\sigma$-field $)$. Let $S_{t}=\sum_{m \leq t} F \circ f^{m}$.

The main result of this paper is the following:

THEOREM 1. The series $\sigma^{2}=\int F^{2} d \mu+2 \sum_{i=1}^{\infty} F \cdot\left(F \circ f^{i}\right) d \mu$ is absolutely convergent, $\int\left(S_{n}\right)^{2} d \mu=n \sigma^{2}+O(1)($ for $n \in \mathbb{N})$ and if $\sigma^{2} \neq 0$, then

(i) $\sup _{r \in R} \mu\left\{S_{n} / \sigma \sqrt{n} \leq r\right\}-(1 / \sqrt{2 \pi}) \int_{-\infty}^{r} e^{-x^{2} / 2} d x \mid=O\left(n^{-\nu}\right)$ for some $\nu>0$;

(ii) without changing its distribution we can redefine the process $\left(S_{t}\right)_{t \geq 0}$ on a richer probabilistic space together with a standard Brownian process $\left(B_{t}\right)_{t \geq 0}$ such that almost surely

for some $\lambda>0$.

$$
\left|\sigma^{-1} S_{t}-B_{t}\right|=O\left(t^{1 / 2-\lambda}\right)
$$

Log- $\log$ laws, integral tests and a weak type of invariance principle follow from this theorem (see [3]). F. Hofbauer and G. Kelle- [3] proved the above theorem in the case of expanding $f$.

\section{Basic technical lemmas}

Let $B:=\bigcup_{n=1}^{\infty} f^{n}(A)$ and $V_{0}$ be an open neighbourhood of $B$. Let $U:=\bigcup_{a \in A} U_{a}$. We can assume that the sets $U_{a}, a \in A$, are disjoint and:

$$
\begin{gathered}
f(U) \subset V_{0} ; \\
\left(f^{-1}(A) \backslash A\right) \cap U=\varnothing .
\end{gathered}
$$


It follows from (v) that $B \backslash A \subset I \backslash U, f(B \backslash A) \subset B \backslash A$ and $B \backslash A$ is closed. So, there exists a neighbourhood $V_{1}$ of the set $B \backslash A$ such that

$$
f\left(V_{1}\right) \cap U=\varnothing \text {. }
$$

Put $V:=V_{0} \cap\left(V_{1} \cup U\right)$.

LEMMA 1. For every $\gamma>1$ there exists $n_{0} \in \mathbb{N}$ such that for all $n \geq n_{0}$ and all $x \in I$ if $f^{n}(x) \notin V$, then $\left|\left(f^{n}\right)^{\prime}(x)\right| \geq \gamma$.

Proof. For all $x \in I$ let $\Delta_{n}(x)$ be the connected component of the set $I \backslash \bigcup_{k=0}^{n-1} f^{-k}(A)$ which contains $x$. By [5, th. 4.6]

$$
\lim _{n \rightarrow \infty}\left(\sup _{y \in I} \lambda\left(\Delta_{n}(y)\right)\right)=0 .
$$

The proof follows by using the argument of W. Szlenk [8, prop. 4].

We remark that by integrating the inequality from condition (vi) we obtain

$$
\alpha|x-a|^{u+1} /(u+1) \leq|f(x)-f(a)| \leqslant \omega|x-a|^{u+1} /(u+1)
$$

for all $x \in U_{a}, a \in A$.

LEMMA 2. Let $f^{i}(a) \in A, f^{i}(x) \in U_{f^{i}(a)}$ for $i=0,1, \ldots, k-1, f^{j+k}(x) \notin U$ for $j=$ $0, \ldots, l-1, f^{l+k}(x) \notin V, k, l \geq 1$. There exists a constant $L>0$ depending only on the size of $U$ and $V$ such that

$$
\left|\left(f^{k+1}\right)^{\prime}(x)\right| \geq \frac{L}{|x-a|} .
$$

Proof. Let $\alpha_{i}, \omega_{i}, u_{i}, \delta_{i}$ be the constants from condition (vi) corresponding to $f^{i}(a)$. Assume that $f^{k}(a) \in A$. Then

$$
\left|f^{k}(a)-f^{k}(x)\right| \geq \operatorname{dist}(A, I \backslash U)=: K_{U}
$$

and by (4)

$$
\left|f^{k-1}(x)-f^{k-1}(a)\right| \geq \frac{\left(u_{k-1}+1\right) K_{U}}{\omega_{k-1}\left|f^{k-1}(x)-f^{k-1}(a)\right|^{u_{k-1}}} .
$$

Hence, using (vi(a)) we obtain

$$
\left|f^{k-1}(x)-f^{k-1}(a)\right| \geq \frac{\left(u_{k-1}+1\right) \alpha_{k-1}}{\omega_{k-1}} \frac{K_{U}}{\left|f^{\prime}\left(f^{k-1}(x)\right)\right|}
$$

Repeating the above argument $k$ times we arrive at the inequality

$$
|x-a| \geq \prod_{i=0}^{k-1} \frac{\left(u_{i}+1\right) \alpha_{i}}{\omega_{i}} \frac{K_{U}}{\prod_{i=0}^{k-1} f^{\prime}\left(f^{i}(x)\right) \mid} .
$$

By $[5$, th. 1.3] there exists a $\nu>1$ and $m \geq 1$ such that

$$
\text { if } f^{j}(x) \notin U \text { for } j=0, \ldots, m-1 \text { then }\left|\left(f^{m}\right)^{\prime}(x)\right| \geq \nu \text {. }
$$

Therefore there exists a constant $c_{0}>0$ depending only on the size of $U$ such that

$$
\left|\left(f^{k+l}\right)^{\prime}(x)\right| \geq \frac{K_{U} c_{0}}{|x-a|} \prod_{i=0}^{k-1} \frac{\left(u_{i}+1\right) \alpha_{i}}{\omega_{i}}
$$

Assume now that $f^{k}(a) \notin A$. By $(v), f^{j+k}(a) \notin U$ for all $j \geq 0$. By assumption 
$f^{l+k}(x) \notin V$, so

$$
\left|f^{l+k}(x)-f^{l+k}(a)\right| \geq \operatorname{dist}(B, I \backslash V)=: K_{V}
$$

Therefore for some $\theta \in\left(f^{k}(x), f^{k}(a)\right)$ we have

$$
\left|\left(f^{l}\right)^{\prime}\left(f^{k}(x)\right)\right| \geq \frac{\left|\left(f^{l}\right)^{\prime}\left(f^{k}(x)\right)\right|}{\left|\left(f^{l}\right)^{\prime}(\theta)\right|} \frac{K_{V}}{\left|f^{k}(x)-f^{k}(a)\right|} .
$$

If $\left[f^{j+k}(a), f^{j+k}(x)\right] \subset I \backslash U$ for $j=0, \ldots, l-1$ then by (5) the same argument as in the case of an expanding $f$ proves that there exists a constant $K>0$ depending only on the size of the neighbourhood $U$ such that

$$
\frac{\left|\left(f^{\prime}\right)^{\prime}\left(f^{k}(x)\right)\right|}{\left|\left(f^{\prime}\right)^{\prime}(\theta)\right|} \leq K \text {. }
$$

So we have

$$
\left|f^{k}(x)-f^{k}(a)\right| \geq \frac{K \cdot K_{V}}{\left|\left(f^{\prime}\right)^{\prime}\left(f^{k}(x)\right)\right|}
$$

But if $\left[f^{j}\left(f^{k}(a)\right), f^{j}\left(f^{k}(x)\right)\right] \cap U \neq \varnothing$ for some $j, 0 \leq j \leq l-1$, then

$$
U_{a_{0}} \subset\left[f^{j}\left(f^{k}(a)\right), f^{j}\left(f^{k}(x)\right)\right]
$$

for some $a_{0} \in A\left(f^{j+k}(a) \notin U\right.$ for $j \geq 0$ in virtue of $\left.(v)\right)$. If $j$ is the smallest such iteration, then

$$
\left|f^{k}(x)-f^{k}(a)\right| \geq \frac{K \cdot K_{U}}{\left|\left(f^{j}\right)^{\prime}\left(f^{k}(x)\right)\right|}
$$

Now using (7) or $\left(7^{\prime}\right)$ (depending on the situation) we proceed as in the case $f^{k}(a) \in A$. The role of the constant $K_{U}$ is now played by

$$
\frac{K \cdot K_{V}}{\left|\left(f^{l}\right)^{\prime}\left(f^{k}(x)\right)\right|} \text { or } \frac{K \cdot K_{U}}{\left|\left(f^{j}\right)^{\prime}\left(f^{k}(x)\right)\right|} \text {. }
$$

As a result we obtain an estimation similar to (6), but the constant $K_{U^{*}} c_{0}$ is now replaced by $K \cdot K_{V}$ or $K \cdot K_{U} c_{0}$. The set $A$ being finite, there are finitely many expressions

$$
\prod_{i=0}^{k-1} \frac{\left(u_{i}+1\right) \alpha_{i}}{\omega_{i}}
$$

Multiplying the smallest of them by the smallest of the numbers $K_{U} \cdot c_{0}, K \cdot K_{V}$, $K \cdot K_{U} \cdot c_{0}$ we obtain $L$.

From lemma 2 and (vi(b)) we derive immediately:

COROLlary 1. There exist constants $E, b>0$ depending only on the size of $U$ and $V$ such that if the assumptions of lemma 2 are satisfied then

(a) $\left|f^{\prime \prime}(x)\right| /\left|f^{\prime}(x) \|\left(f^{k+l}\right)^{\prime}(x)\right| \leq K$,

(b) $\left|\left(f^{k+l}\right)^{\prime}(x)\right| \geq b$.

Notice that in view of (iv) we can choose $U_{a}$ such that if $a \in A$ is periodic with period $p$, then for all $x \in U_{a}$

$$
\left|\left(f^{p}\right)^{\prime}(x)\right| \geq \beta>1
$$


LEMMA 3. If the assumptions of lemma 2 are fulfilled then there exists a constant $H_{0}>0$ depending only on the size of $U$ and $V$ such that

$$
S_{k+l}(x):=\sum_{i=0}^{k+l-1} \frac{\left|f^{\prime \prime}\left(f^{i}(x)\right)\right|}{\left|f^{\prime}\left(f^{i}(x)\right)\right|\left|\left(f^{k+l-i}\right)^{\prime}\left(f^{i}(x)\right)\right|} \leq H_{0} .
$$

Proof. Suppose the assumptions of lemma 2 are satisfied. If $a \in A$ is periodic there is nothing to do in view of (i), (8) and (5). If $a$ is not periodic then $k \leq k_{0}$ for some $k_{0} \in \mathbb{N}$, because $A$ is finite. In view of corollary 1 the sum of the first $k$ terms of the sum $S_{k+1}(x)$ does not exceed $k_{0} \cdot E$. By (5) and (i) the sum of remaining terms does not exceed (up to a constant) the sum of a geometric series with the quotient $1 / \nu$.

Notice that we can make the sets $U_{a}$ so small that

$$
f\left(U_{a}\right) \cap\left(\bigcup_{\substack{b \neq f(a) \\ b \in A}} U_{b}\right)=\varnothing \quad \text { for all } a \in A .
$$

LeMMA 4. There exists $H>0$ depending only on the size of $U$ and $V$ such that if $f^{n}(y) \notin V$, then

$$
\left|\left(\frac{1}{\left(f^{n}\right)^{\prime}(y)}\right)^{\prime}\right| \leq S_{n}(y) \leq H .
$$

Proof. Suppose $f^{n}(y) \notin V$. On account of (1), (3) and (9) we can divide the sequence $y, f(y), \ldots, f^{n}(y)$ (except for the first $q_{0} \geq 0$ terms not belonging to $U$ ) into blocks satisfying the assumptions of lemma 2 .

Let $\gamma>1$ and $n_{0} \in \mathbb{N}$ be as in lemma 1. Suppose that we have $m \cdot n_{0}+r(m, r \in$ $\left.\mathbb{N} \cup\{0\}, r<n_{0}\right)$ blocks in the sequence $y, f(y), \ldots, f^{n}(y)$. In view of (5) there exists $H_{1}>0$ depending only on the size of $U$ such that $S_{q_{0}}(y) \leq H_{1}$. Hence, the desired estimate is obtained by using lemma 1 , corollary $1(\mathrm{~b})$ and lemma 3 .

LEMMA 5. There exist $c>0, \theta>1$ depending only on the size of $U$ and $V$ such that if the assumptions of lemma 2 are satisfied then $\left|\left(f^{k+l}\right)^{\prime}(x)\right| \geq c \cdot \theta^{k+l}$.

Proof. If $a$ is periodic there is nothing to do by (8) and (5).

Suppose $a$ is not periodic. Let $\alpha_{i}, \omega_{i}, u_{i}$ be the constants from condition (vi) corresponding to $f^{i}(a)$. Using the inequalities (4) $k$ times we arrive at

$$
\begin{aligned}
& \left|f^{k}(x)-f^{k}(a)\right| \\
& \quad \geq \frac{\alpha_{k-1}}{u_{k-1}+1}\left(\frac{\alpha_{k-2}}{u_{k-2}+1}\right)^{\left(u_{k-1}+1\right)} \cdots\left(\frac{\alpha}{u+1}\right)^{\left(u_{k-1}+1\right) \cdots\left(u_{1}+1\right)}|x-a|^{\left(u_{k-1}+1\right) \cdots(u+1)} .
\end{aligned}
$$

But $k \leq k_{0}$ (cf. the proof of lemma 3 ), so there exist $c_{3}>0, q>0$ depending only on the size of $U$ such that

$$
\left|f^{k}(x)-f^{k}(a)\right| \geq c_{3}|x-a|^{q}
$$

Let $j$ be the greatest integer such that $\left[f^{i+k}(x), f^{i+k}(a)\right] \cap U=\varnothing$ for $i=0, \ldots, j-1$. By (5) there exists a constant $c_{4}>0$ depending only on the size of $U$ such that

$$
|I| \geq f^{j+k}(x)-f^{j+k}(a)\left|\geq c_{4} \nu^{j / m}\right| f^{k}(x)-f^{k}(a) \mid .
$$


The last inequalities together with (11) give

$$
|x-a| \leq \frac{\sqrt[q]{|I|}}{c_{3} \sqrt[g]{c_{4}}} \frac{1}{(\sqrt[q]{\nu})^{j}}
$$

The same argument as in the proof of lemma 2 shows that

$$
\left|\left(f^{k+j}\right)^{\prime}(x)\right| \geq L_{1} /|x-a|
$$

where $L_{1}>0$ depends only on $U$ and $V$.

By (12) and (13) there exists $c_{5}>0$ depending only on $U$ and $V$ such that

$$
\left|\left(f^{k+j}\right)^{\prime}(x)\right| \geq c_{5}(\sqrt[a]{\nu})^{j+k}
$$

Now the desired estimate follows from the last inequality and (5).

LEMMA 6. There exist constants $G>0, \nu>1$, depending only on $U$ and $V$ such that if $f^{n}(y) \notin V$, then $\left|\left(f^{n}\right)^{\prime}(y)\right| \geq G \cdot \nu^{n}$.

Proof. If $f^{n}(y) \notin V$, then the sequence $y, f(y), \ldots, f^{n}(y)$ (except the first $q_{0} \geq 0$ terms not belonging to $U$ ) can be divided into $k$ blocks each of which satisfies the assumptions of lemma 2 . Hence by lemma 5

$$
\left|\left(f^{n}\right)^{\prime}(y)\right| \geq b_{0} c^{k} \theta^{n}
$$

where $b_{0}>0$ depends only on $U$.

If $c \geq 1$, we obtain the desired inequality. Suppose that $c<1$. Let $n_{1} \in \mathbb{N}$ be such that $c \cdot \theta^{n_{1} / 2}>1$. If $k<n / n_{1}$ then by (14),

$$
\begin{aligned}
\left|\left(f^{n}\right)^{\prime}(y)\right| & \geq b_{0} c^{n / n_{1}}\left(\theta^{n_{1}}\right)^{n / n_{1}} \\
& =b_{0}\left[\left(c \cdot \theta^{n_{1} / 2}\right)^{1 / n_{1}}\left(\theta^{n_{1} / 2}\right)^{1 / n_{1}}\right]^{n} \\
& >b_{0}(\sqrt{\theta})^{n} .
\end{aligned}
$$

If $k>n / n_{1}$, we have $k=\ln _{0}+r, 0 \leq r<n_{0}$, where $n_{0}$ is as in lemma 1 , and

$$
\left|\left(f^{n}\right)^{\prime}(y)\right| \geq b_{0} \gamma^{\prime} c^{r} \geq b_{0} c^{n_{0}} \gamma^{l} .
$$

By assumption $k>n / n_{1}$, so $l=(k-r) / n_{0}>\left(n / n_{1}-r\right) / n_{0}>\left(n / n_{1} n_{0}\right)-1$. From (15) and the above estimate we obtain

$$
\left|\left(f^{n}\right)^{\prime}(y)\right| \geq \frac{b_{0} c^{n_{0}}}{\gamma}\left(\gamma^{1 / n_{0} n_{1}}\right)^{n}
$$

3. Estimation of the diameter of $\bigvee_{0}^{n-1} f^{-i} \mathscr{A}$ in the sense of Lebesgue measure Let $\mathscr{A}$ be the partition of $I$ given by the points of $A$. We will show that the number $\max _{\Delta \in V_{0}^{n-1} f^{-i} \mathscr{A}} \lambda(\Delta)$ decreases exponentially with respect to $n$. We will call this number the diameter of $V_{0}^{n-1} f^{-i} \mathscr{A}$ in the sense of Lebesgue measure.

Lemma 7. Let $\Delta=(v, w) \in \bigvee_{0}^{n-1} f^{-i} \mathscr{A}, \Delta \notin \bigvee_{0}^{n} f^{-i} \mathscr{A}$ and $l$ be the smallest iteration such that $f^{\prime}(w) \in A$. There exists $z \in(v, w)$ such that

(a) $\left|\left(f^{n}\right)^{\prime}(z)\right| \geq D \nu^{n}$;

(b) for some $n_{z} \geq n-l$ the sequence $f^{l}(z), f^{l+1}(z), \ldots, f^{l+n_{z}}(z)$ satisfies the assumptions of lemma 2 and

$$
\left|f^{j+l}(z)-f^{j+l}(w)\right|<K_{V} \quad \text { for } j=0, \ldots, n_{z}-1,
$$


where the constant $D>0$ depends only on $U$ and $V, \nu$ is as in lemma 6 and $K_{V}$ as in the proof of lemma 2.

Proof. We can assume that $n$ is the biggest natural number such that $\Delta=(v, w) \in$ $V_{0}^{n-1} f^{-1} \mathscr{A}$ (such an $n$ exists because of the lack of homtervals). Hence

$$
f^{n}(\Delta) \cap A \neq \varnothing \text {. }
$$

Let $l$ be the smallest iteration such that $f^{\prime}(w)=b \in A$. Write $x=f^{\prime}(v)$.

Suppose that $x \in U_{b}$. The ends of the interval $f^{n}(\Delta)$ belong to $B$, so in view of (16) there exists $y \in[x, b)$ (or $(b, x])$ such that the sequence $y, f(y), \ldots, f^{n-1}(y)$ satisfies the assumptions of lemma 2 (with $b$ instead of $a$ ) and

$$
\left|f^{j}(y)-f^{j}(b)\right|<K_{V} \quad \text { for } j=0, \ldots, n-l-1 .
$$

Therefore by lemma 5

$$
\left|\left(f^{n-l}\right)^{\prime}(y)\right| \geq c \theta^{n-1} .
$$

Let $z \in(v, w)$ be such that $f^{\prime}(z)=y$. It is easy to show by using (2), (9) and the monotonicity of $f$ on the components of $I \backslash A$, that $f^{l-1}(z) \notin U$. Hence by (3) and the definition of $V$ we have $f^{l-1}(z) \notin V$ and we can use lemma 6 to estimate $\left|\left(f^{l}\right)^{\prime}(z)\right|$ by $\nu^{\prime}$ up to a constant depending only on $U$ and $V$. This estimate together with (18) gives

$$
\left|\left(f^{n}\right)^{\prime}(z)\right| \geq R_{1} \nu^{n}
$$

where $R_{1}>0$ is a constant depending only on $U$ and $V$.

Let us now suppose $x \notin U_{b}$ and let $y \in(x, b)$ be the centre of the interval $U_{b} \cap(x, b)$ (or $U_{b} \cap(b, x)$ ). Denote by $n_{b}$ the smallest iteration such that the sequence $y, f(y), \ldots, f^{n_{b}}(y)$ satisfies the assumptions of lemma 2 and $\left|f^{j}(y)-f^{j}(b)\right|<K_{V}$ for $j=0, \ldots, n_{b}-1$.

If $n_{b}<n-l$, then there exists $y^{\prime} \in U_{b} \cap(x, b)$ (or $U_{b} \cap(b, x)$ ) such that the sequence $y^{\prime}, f\left(y^{\prime}\right), \ldots, f^{n-1}\left(y^{\prime}\right)$ satisfies the assumptions of lemma 2 and

$$
\left|f^{j}\left(y^{\prime}\right)-f^{j}(b)\right|<K_{V} \quad \text { for } j=0, \ldots, n-l-1 .
$$

Let $z^{\prime} \in(v, w)$ be such that $f^{\prime}\left(z^{\prime}\right)=y^{\prime}$. In exactly the same way as for $z$, we prove that the estimate (19) remains true for $z^{\prime}$.

If $n_{b} \geq n-l$ then

$$
\left|\left(f^{n_{b}}\right)^{\prime}(y)\right|=\left|\left(f^{n_{b}-n-l}\right)^{\prime}\left(f^{n-l}(y)\right)\right|\left|\left(f^{n-l}\right)^{\prime}(y)\right|,
$$

where the first factor on the right side of the above equality is bounded from above by a constant $K_{0}$ depending on $U$ because the set of numbers $n_{b}, b \in A$, is finite. This argument together with lemma 5 gives

$$
\left|\left(f^{n-l}\right)^{\prime}(y)\right| \geq \frac{c}{K_{0}} \theta^{n-l} .
$$

If $z \in(v, w)$ is such that $f^{l}(z)=y$, then as above we obtain the estimate analogous to $(19)$ :

$$
\left|\left(f^{n}\right)^{\prime}(z)\right| \geq \frac{R_{1}}{K_{0}} \nu^{n}
$$


Remark. We can formulate and prove in a similar way a lemma analogous to lemma 7 for the left end point of $\Delta$.

THEOREM 2. There exists $d>0$ such that for $n \in \mathbb{N}, \max _{\Delta \in \mathrm{V}_{0}^{n-1} f^{-i} \oiint} \lambda(\Delta) \leq d / \nu^{n}$.

Proof. We can assume than $n$ is the biggest number such that $\Delta \in \bigvee_{0}^{n-1} f^{-i} \mathscr{A}$. Let $\Delta=(v, w), z$ and $n_{z}$ be as in lemma 7. Write $y=f^{\prime}(z), b=f^{\prime}(w)$. For all $m \geq n_{z}$ we define $I_{m}=\left\{x \in(y, b):\left|f^{j}(x)-f^{j}(b)\right|<K_{V}\right.$ for $j=0, \ldots, m-1$ and $\left|f^{m}(x)-f^{m}(b)\right| \geq$ $\left.K_{V}\right\}$. It is easy to see that $I_{m}$ is an interval and $\left.f^{m}\right|_{I_{m}}$ is monotonic. Hence, by using lemma 5, we obtain

$$
\lambda\left(I_{m}\right) \leq \lambda(I) / c \theta^{m} .
$$

Observe that by lemma $7(\mathrm{~b})(y, b)=\bigcup_{m=n_{z}}^{\infty} I_{m}$. Therefore

$$
\lambda((y, b)) \leq \sum_{m=n_{z}}^{\infty} \lambda\left(I_{m}\right) \leq \frac{\theta \lambda(I)}{c(\theta-1)} \frac{1}{\theta^{n-I}}
$$

Now, using the standard argument we prove that for all $t \in(z, w)\left|\left(f^{l}\right)^{\prime}(t)\right|$ can be estimated from below by $\nu^{l}$, up to a constant depending only on $U$ and $V$. From this estimate and (20) we obtain

$$
\lambda((z, w)) \leq R_{2}\left(1 / \nu^{n}\right),
$$

where $R_{1}>0$ depends only on $U$ and $V$. Now using the version of lemma 7 for the left end point of $\Delta$ we find a point $u \in \Delta$ such that

$$
\lambda((v, u)) \leq R_{2}\left(1 / \nu^{n}\right)
$$

and by lemma $7(a)$ and its version for the left end point of $(u, z)$ we get, if we observe that $\left|f^{\prime}\right|$ has no positive local minima:

$$
\lambda((u, z)) \leq \frac{\lambda(I)}{D} \frac{1}{\nu^{n}}
$$

from (21), (22) and (23) we obtain the desired estimate for $\lambda(\Delta)$.

Definition 1 . If $m$ is a measure on $I$ then we define the diameter of a partition $\mathscr{b}$ as $\max _{C \in \mathscr{C}} m(C)$.

Theorem 2 says that the diameter of $V_{0}^{n-1} f^{-i} \mathscr{A}$ in the sense of $\lambda$ decreases exponentially with $n$.

4. Estimation of the diameter of $V_{0}^{n-1} f^{-i} \mathscr{A}$ in the sense of the invariant measure The exponential rate of decrease of the diameters of $\bigvee_{0}^{n-1} f^{-i} \mathscr{A}$ in the sense of the invariant measure $\mu$ follows from theorem 2 and the following:

THEOREM 3. If $\lambda(G)$ is sufficiently small then $\mu(G) \leq R \sqrt[s]{\lambda(G)}$ for $R>0, s>1$ depending only on $U$ and $V$.

Proof. Let $m_{0} \geq 1$ be such that $f^{m_{0}}$ satisfies the conditions (i)-(vi) and (vii), (viii) [5]:

(vii) $\left|\left(f^{m_{0}}\right)^{\prime}\right|>1$ on $U\left(f^{m_{0}}\right)\left(U\left(f^{m_{0}}\right)=\bigcup_{i=0}^{m_{0}-1} f^{-i}(U)\right)$;

(viii) If $a \in A\left(f^{m_{0}}\right)$ is a periodic point for $f^{m_{0}}$, then it is a fixed point for $f^{m_{0}}$.

Such an $m_{0}$ exists - see lemma 3.1 of [5].

Let $E_{n}=\left\{x \in I: f^{k m_{0}}(x) \notin U\left(f^{m_{0}}\right), k=0, \ldots, n-1\right\} . E_{n}$ is a neighbourhood of $B \backslash A\left(f^{m_{0}}\right)$ and it is proved in [5] (the proof of lemma 3.7) that there are constants 
$d_{1}>0, \xi_{1}, \xi_{2} \in(0,1)$ such that for every $n \in \mathbb{N}$

$$
\sup _{k \in N} \int_{E_{n}} f_{*}^{k m_{0}}(1) d \lambda \leq \sum_{l=n}^{\infty}(l+1) d_{1} \xi_{1}^{l}+\lambda(I) \xi_{2}^{n} .
$$

Let $a$ be an end point of a component of $E_{n}$ and $b \in B$ a point from this component. For some $k \leq n-1$ we have $f^{k m_{0}}(a) \in U\left(f^{m_{0}}\right)$ and

$$
\left|f^{k m_{0}}(a)-f^{k m_{0}}(b)\right| \geq \operatorname{dist}\left(B \backslash A\left(f^{m_{0}}\right), U\right)=: h_{B} \text {. }
$$

Hence

$$
|a-b| \geq \frac{h_{B}}{F_{0}^{k}} \geq \frac{h_{B}}{F_{0}^{n}}
$$

where $F_{0}=\sup _{x \in I}\left|\left(f^{m_{0}}\right)^{\prime}(x)\right|$. Since the component of $E_{n}$ and the point $b$ are arbitrary, we have shown

$$
\operatorname{dist}\left(B \backslash A\left(f^{m_{0}}\right), I \backslash E_{n}\right) \geq \frac{h_{B}}{F_{0}^{n}} .
$$

Let $A_{1}\left(f^{m_{0}}\right)$ denote the set of periodic points of the set $A\left(f^{m_{0}}\right)$. For $a \in A_{1}\left(f^{m_{0}}\right)$ we define

$$
V_{n}(a)=\left\{x \in I: f^{m_{0} i}(x) \in U_{a} \text { for } i=0, \ldots, n-1\right\},
$$

as in the proof of lemma 3.6 of [5]. It is shown in this proof that there exist $d_{2}>0$, $\xi_{3}, \xi_{4} \in(0,1)$ such that

$$
\sup _{k \in N} \int_{V_{n}(a)} f_{*}^{k m_{0}}(1) d \lambda \leq d_{2} \sum_{i=n}^{\infty} \xi_{3}^{l}+\lambda(I) \xi_{4}^{n}
$$

Write $V_{n}=\bigcup_{a \in A_{1}\left(f^{m_{0}}\right)} V_{n}(a), h_{A}=\operatorname{dist}\left(A_{1}\left(f^{m_{0}}\right), I \backslash U\left(f^{m_{0}}\right)\right)$. As in (25) we prove that

$$
\operatorname{dist}\left(A_{1}\left(f^{m_{0}}\right), I \backslash V_{n}\right) \geq h_{A} / F_{0}^{n} \text {. }
$$

Denote by $A_{2}\left(f^{m_{0}}\right)$ the set of non-periodic points of $A\left(f^{m_{0}}\right)$. The set $A_{2}\left(f^{m_{0}}\right)$ is finite so it is easy to construct a neighbourhood $G_{n}$ of $A_{2}\left(f^{m_{0}}\right)$ being a finite sum of intervals such that

$$
\operatorname{dist}\left(A_{2}\left(f^{m_{0}}\right), I \backslash G_{n}\right)=h_{A} / F_{0}^{n} \text {. }
$$

From [5, (3.4)] there are $d_{3}>0, \xi_{5} \in(0,1)$ such that

$$
\sup _{k \in N} \int_{G_{n}} f_{*}^{k m_{0}}(1) d \lambda \leq d_{3}\left(\frac{h_{A}}{F_{0}^{n}}\right)^{1-\xi_{5}} \text {. }
$$

Put $W_{n}=E_{n} \cup V_{n} \cup G_{n}$. If $n$ is sufficiently large then the sets $E_{n}, V_{n}$ and $G_{n}$ are disjoint and by (25), (27) and (28) we have for $h \leq h_{A}, h_{B}$

$$
\operatorname{dist}\left(B, I \backslash W_{n}\right) \geq h / F_{0}^{n} \text {. }
$$

The estimates (29), (26) and (24) imply the existence of $P_{0}>0$ and $\xi \in(0,1)$ such that

$$
\sup _{k \leq 0} \int_{W_{n}} f_{*}^{k m_{0}}(1) d \lambda \leq P_{0} \xi^{n}
$$

Fix $\varepsilon>0$. If we take $n=\left[\log _{F_{0}}\left(\varepsilon / 3 P_{0}\right) / \log _{F_{0}}(\xi)\right]+1$, then

$$
P_{0} \xi^{n} \leq \varepsilon / 3
$$


and

$$
\frac{h}{F_{0}^{n}} \geq \frac{h}{F_{0}} \cdot\left(\frac{\varepsilon}{3 P_{0}}\right)^{-1 / \log _{F_{0}}(\xi)}
$$

Now we can follow the proof of [5, prop. 3.8] with $W_{n}$ instead of $W .[5,3.10]$ is satisfied because of (31) and (32). Following the proof we find a $\delta$ which satisfies

$$
\delta \geq d_{4} \varepsilon \operatorname{dist}\left(B, I \backslash W_{n}\right),
$$

where $d_{4}$ depends only on $U$ and is such that

$$
\text { if } \lambda(G)<\delta, \text { then } \int_{G} f_{*}^{n}(1) d \lambda \leq \varepsilon \text { for all } n \in \mathbb{N} \text {. }
$$

But in view of (30), (33) and (34)

$$
\delta \geq d_{5} \varepsilon^{1+\sigma}
$$

for some $d_{5}>0, \sigma>0$ which do not depend on $\delta$ and $\varepsilon$. Now the theorem follows from $(35),(36)$ and $[5$, th. $6.2(\mathrm{e})]$.

\section{Estimation of the rate of decrease of the mixing coefficient}

Definition 2. For two partitions $\mathscr{C}, \tilde{\mathscr{C}}$ of $I$ we define

$$
D(\mathscr{C}, \tilde{\mathscr{C}})=\sum_{C \in \mathscr{C}} \sum_{\tilde{C} \in \tilde{\mathscr{C}}}|\mu(C \cap \tilde{C})-\mu(C) \mu(\tilde{C})| .
$$

We will show that $D\left(\bigvee_{0}^{n-1} f^{-i} \mathscr{A}, \bigvee_{n+l}^{n+l+k-1} f^{-i} \mathscr{A}\right)$ decreases exponentially with respect to $l$ uniformly in $n$ and $k$.

Let $F=\sup _{x \in I}\left|f^{\prime}(x)\right|$ and take $p_{0} \in \mathbb{N}$ such that $\sqrt[p_{0}]{F}<\nu$, where $\nu$ is as in theorem 2 .

Definition 3. Fix $M \in \mathbb{N}$. For every $m \in \mathbb{N} \cup\{0\}$ we define:

(a) $\mathscr{D}_{m, M}^{0}$ to be the set of all atoms of $V_{0}^{m+\left[M / p_{0}\right]-1} f^{-i} \mathscr{A}$ whose end points and their $\boldsymbol{m}-1$ consecutive images do not belong to $A$;

(b) $\mathscr{D}_{m, M}$ to be the set of all atoms of $V_{0}^{m+M-1} f^{-i} \mathscr{A}$ which are subsets of the atoms belonging to $\mathscr{D}_{m, M}^{0}$.

Write $h_{n}(y)=\left(1 /\left(f^{n}\right)^{\prime}(y)\right)$ for $n \in \mathbb{N}, y \in I$.

LEMMA 8. The function $h_{n}$ is monotonic on the elements of the partition $\bigvee_{0}^{n-1} f^{-i} \mathscr{A}$.

Proof. In view of (iii) $1 / \sqrt{\left|\left(f^{n}\right)^{\prime}\right|}$ is convex on the elements of $\bigvee_{0}^{n-1} f^{-i} \mathscr{A}$ (see [5, (iii), (iii')]). The square of a non-negative convex function is a convex function, hence $1 /\left|\left(f^{n}\right)^{\prime}\right|$ is convex on the elements of $V_{0}^{n-1} f^{-i} \mathscr{A}$. This proves the assertion of the lemma.

LEMMA 9. Let $\Delta \in \mathscr{D}_{m, M}^{0}$. For every $y \in \Delta,\left|h_{m}(y)\right| \leq H\left(\sqrt[P_{0}]{F}\right)^{M}$, where $H$ is the constant of lemma 4.

Proof. Fix $\Delta \in \mathscr{D}_{m, M}^{0}$. By the definition of $\mathscr{D}_{m, M}^{0} h_{m}$ is finite on all $\bar{\Delta}$. In view of lemma $\left.8 h_{m}\right|_{\bar{\Delta}}$ assumes its greatest value at one of the end points of $\Delta$, denote it by $z$. Now it suffices to estimate $S_{m}(z)$, because $\left|h_{m}(z)\right| \leq S_{m}(z)$.

Let $r$ be the smallest positive integer such that $f^{m+r}(z) \in A$ (it is clear that $\left.r<\left[M / p_{0}\right]\right)$. By (2), (3) and the definition of $V, f^{m+r-1}(z) \notin V$. Therefore by lemma 4 
$S_{m+r-1}(z) \leq H$. On the other hand

$$
\begin{aligned}
S_{m+r-1}(z) & =S_{m}(z) \frac{1}{\left|\left(f^{r-1}\right)^{\prime}\left(f^{m}(z)\right)\right|}+S_{r-1}\left(f^{m}(z)\right) \\
& \geq S_{m}(z) \frac{1}{F^{M / p_{0}}}
\end{aligned}
$$

Therefore $S_{m}(z) \leq H\left(\sqrt[p_{0}]{F}\right)^{M}$.

LEMMA 10. Let $\Delta \in \mathscr{D}_{m, M}, y^{\prime}, y^{\prime \prime} \in \Delta$. If $M$ is sufficiently large, then

$$
\left|\frac{\left(f^{m}\right)^{\prime}\left(y^{\prime \prime}\right)}{\left(f^{m}\right)^{\prime}\left(y^{\prime}\right)}-1\right| \leq 2 H\left(\sqrt[p_{0}]{F}\right)^{M}\left|f^{m}\left(y^{\prime}\right)-f^{m}\left(y^{\prime \prime}\right)\right| .
$$

Proof. Let $\Delta \in \mathscr{D}_{m, M}, y^{\prime}, y^{\prime \prime} \in \Delta$. By definition $\Delta$ is contained in an atom of $\mathscr{D}_{m, M}^{0}$, so we can use lemma 9 to obtain

$$
\left|\frac{1}{\left(f^{m}\right)^{\prime}\left(y^{\prime}\right)}-\frac{1}{\left(f^{m}\right)^{\prime}\left(y^{\prime \prime}\right)}\right| \leq H\left(\sqrt[p_{0}]{F}\right)^{M}\left|y^{\prime}-y^{\prime \prime}\right| .
$$

The function $\mid\left(f^{m}\right)^{\prime} \|_{\left[y^{\prime}, y^{\prime \prime}\right]}$ assumes its minimum at one of the ends points of $\left[y^{\prime}, y^{\prime \prime}\right]$ (by (iii) and the definition of $\mathscr{D}_{m, M}$ ), say at $y^{\prime \prime}$. Hence

$$
\left|\frac{\left(f^{m}\right)^{\prime}\left(y^{\prime \prime}\right)}{\left(f^{m}\right)^{\prime}\left(y^{\prime}\right)}-1\right| \leq H\left(\sqrt[p_{0}]{F}\right)^{M}\left|f^{m}\left(y^{\prime}\right)-f^{m}\left(y^{\prime \prime}\right)\right|
$$

If $\left|\left(f^{m}\right)^{\prime}\right|$ has its minimum at $y^{\prime}$ we obtain just an analogous estimation of $\left|\left[\left(f^{m}\right)^{\prime}\left(y^{\prime}\right) /\left(f^{m}\right)^{\prime}\left(y^{\prime \prime}\right)\right]-1\right|$. In this case, if $M$ is sufficiently large, then the right side of this inequality does not exceed $\frac{1}{2}$ (because of theorem 2 and the choice of $p_{0}$ such that $\sqrt[p_{0}]{F}<\nu$ ). Now it is easy to obtain the desired inequality.

Let $m_{0}$ be such that $f^{m_{0}}$ satisfies (i)-(vi) and (vii), (viii). Denote by $A^{\prime}\left(f^{m_{0}}\right)$ the set of the points from $A\left(f^{m_{0}}\right)$ and the ends points of the components of $U\left(f^{m_{0}}\right)$. Let $\mathscr{A}^{\prime}$ be a partition defined by the points of $A^{\prime}\left(f^{m_{0}}\right)$. Let $W_{n}$, for sufficiently large $n \in \mathbb{N}$, be the neighbourhood of $B$ defined in the proof of theorem 3. By the definition of $W_{n}$ :

(37) $\mathrm{W}_{\mathrm{n}}$ is a sum of some atoms belonging to $\bigvee_{0}^{n m_{0}-1} f^{-i}\left(\mathscr{A}^{\prime}\right)$ and of $G_{n}$ (which is a finite sum of intervals).

Definition 4. Fix a large $M \in \mathbb{N}$. For every $m \in \mathbb{N} \cup\{0\}$ we define a family $\mathscr{A}_{m, M}$ of good atoms of $\bigvee_{0}^{m+M-1} f^{-i}\left(\mathscr{A}^{\prime}\right)$ as a family of all $\Delta \in \bigvee_{0}^{m+M-1} f^{-i} \mathscr{A}^{\prime}$ such that

(a) $\Delta \subset \operatorname{supp} \mu$;

(b) $\Delta$ is contained in an atom belonging to $\mathscr{D}_{m, M}$;

(c) the end points of $\Delta$ and their $m-1$ consecutive images do not belong to $A^{\prime}\left(f^{m_{0}}\right)$;

(d) $\left(\Delta \cup f^{m}(\Delta)\right) \cap W_{\left[M / 2 p_{0} m_{0}\right]}=\varnothing$.

Let $\varphi$ be the density of $\mu$ with respect to the Lebesgue measure $\lambda$. B. Szewc proved in [7] that if $f$ satisfies (i)-(vi), then $\varphi \in C^{2}$ and for $j=0,1,2, x \in I \backslash B$

$$
\left|\varphi^{(j)}(x)\right| \leq \sum_{n=0}^{\infty} \sum_{\substack{a \in A \\ f(a) \notin A}} \frac{1}{\left|\left(f^{n}\right)^{\prime}(f(a))\right|^{\eta(a)}} \frac{1}{\left|x-f^{n+1}(a)\right|^{\xi(a)+j}}+\text { ! }
$$


where $\xi(a)=u_{a} /\left(u_{a}+1\right)\left(u_{a}\right.$ is the constant from condition (vi) corresponding to the point $a), \eta(a)=1-\xi(a)$

Lemma 11. There exist $L_{0}>0, \xi_{0} \in(0,1)$ such that if $x \in I \backslash B$, then for $j=0,1,2$

$$
\left|\varphi^{(j)}(x)\right| \leq L_{0} /|x-b|^{\xi_{0}+j},
$$

where $b$ is the point of $B$ nearest to $x$.

Proof. It is a simple consequence of (38) and (5).

LEMMA 12. There exist $Z>0, \zeta \in(0,1)$ such that if $\Delta \in \mathscr{A}_{m, M}, m \in \mathbb{N} \cup\{0\}, x, y \in \Delta$, then

$$
\left|\frac{\varphi(x)}{\varphi(y)}-1\right| \leq Z \zeta^{M} \text {. }
$$

Proof. Fix $\Delta \in \mathscr{A}_{m, M}$. By definition $\Delta$ is contained in the complement of a neighbourhood of singularities of $\varphi$ and in $\operatorname{supp} \varphi$. Hence $\varphi_{\bar{\Delta}}$ is positive, convex ([5]), and

$$
\sup _{x \in \Delta}\left|\varphi^{\prime}(x)\right|=\left|\varphi^{\prime}(v)\right|,
$$

where $v$ is one of the end points of $\Delta$. So we have

$$
|\varphi(x)-\varphi(y)| \leq\left|\varphi^{\prime}(v)\right||x-y| \quad \text { for } x, y \in \Delta,
$$

and by definition 4 and theorem 2 ,

$$
|\varphi(x)-\varphi(y)| \leq d\left|\varphi^{\prime}(v)\right| / \nu^{M} .
$$

In view of lemma 11

$$
\left|\varphi^{\prime}(v)\right| \leq \frac{L_{0}}{|v-b|^{\xi_{0}+1}}, \quad b \in B .
$$

By definition 4, $\Delta \cap W_{\left[M / 2 p_{0} m_{0}\right]}=\varnothing$, so using (30) we obtain

$$
|v-b| \geq \operatorname{dist}\left(I \backslash W_{\left[M / 2 p_{0} m_{0}\right]}, B\right) \geq \frac{h}{F^{M / 2 p_{0}}} .
$$

From the last estimate, (40) and (39) it follows that

$$
|\varphi(x)-\varphi(y)| \leq \frac{d L_{0}}{h^{\xi_{0}+1}}\left(\frac{p_{0}}{\nu}\right)^{M},
$$

which ends the proof because $\varphi$ is separated from zero by a positive constant on supp $\varphi$.

The next lemma is analogous to [3, lemma 12].

Lemma 13. There exist $S>0, \Omega>1$ such that if $M \in \mathbb{N}$ is sufficiently large, $m \in \mathbb{N} \cup\{0\}$, then:

(a) for every $\Delta \in \mathscr{A}_{m, M}, f^{m}(\Delta) \in \mathscr{A}_{0, M}$;

(b) $\mu\left(\bigcup \mathscr{A}_{m, M}\right)>1-\left(S / \Omega^{M}\right)$;

(c) if $\tilde{\Delta}$ is a measurable subset of $\Delta \in \mathscr{A}_{m, M}$ then

$$
\left|\frac{\mu\left(f^{m}(\tilde{\Delta})\right)}{\mu\left(f^{m}(\Delta)\right)}-\frac{\mu(\tilde{\Delta})}{\mu(\Delta)}\right|<\frac{S}{\Omega^{M}} \frac{\mu(\tilde{\Delta})}{\mu(\Delta)} .
$$

Proof. $(a)$ is a consequence of definition 4(c). For a proof of (b) let us notice that for $\Delta \in \bigvee_{0}^{m+M-1} f^{-i} \mathscr{A}^{\prime}, \Delta \notin \mathscr{A}_{m, M}$ if and only if it satisfies one of the following four 
conditions:

(1) $\Delta \cap(I \backslash \operatorname{supp} \mu) \neq \varnothing$;

(2) $\Delta$ is not contained in an atom in $\mathscr{D}_{m, M}$;

(3) there exists $k, 0 \leq k<m$ such that

$$
\overline{f^{k}(\Delta)} \cap A^{\prime}\left(f^{m_{0}}\right) \neq \varnothing
$$

(4) $\left(\Delta \cup f^{m}(\Delta)\right) \cap W_{\left[M / 2 p_{0} m_{0}\right]} \neq \varnothing$.

There are only two atoms in $\bigvee_{0}^{m+M-1} f^{-i} \mathscr{A}^{\prime}$ with a non-zero $\mu$-measure which satisfy the first condition. This follows from the fact that supp $\mu$ is an interval (remark 5.6 and theorem 6.2 (b) of [5]). Hence a suitable estimate of the sum of atoms satisfying condition (1) is an immediate consequence of theorems 2 and 3.

A suitable estimate of the sum of atoms satisfying condition (2) or (3) can be obtained by using theorems 2, 3 and a standard argument (see the proof in [1]).

A suitable estimate of the sum of atoms satisfying condition (4) follows from (37), (31) and theorems 2 and 3.

For a proof of (c) take a measurable subset $\tilde{\Delta}$ of an atom $\Delta \in \mathscr{A}_{m, M}$ and choose a point $x_{0} \in \tilde{\Delta}$. We have

$$
\frac{\mu\left(f^{m}(\tilde{\Delta})\right)}{\mu\left(f^{m}(\Delta)\right)}=\frac{\int_{\tilde{\Delta}} \frac{\varphi\left(f^{m}(x)\right)}{\varphi\left(f^{m}\left(x_{0}\right)\right)} \frac{\left|\left(f^{m}\right)^{\prime}(x)\right|}{\left|\left(f^{m}\right)^{\prime}\left(x_{0}\right)\right|} \frac{\varphi\left(x_{0}\right)}{\varphi(x)} d \mu(x)}{\int_{\Delta} \frac{\varphi\left(f^{m}(x)\right)}{\varphi\left(f^{m}\left(x_{0}\right)\right)} \frac{\left|\left(f^{m}\right)^{\prime}(x)\right|}{\left(f^{m}\right)^{\prime}\left(x_{0}\right) \mid} \frac{\varphi\left(x_{0}\right)}{\varphi(x)} d \mu(x)}
$$

Now we obtain the desired estimate by using lemmas 12 and 10 . If the constants obtained in the proofs of (b) and (c) are different, we may change them to obtain the estimates with the same constants, which we will call $S>0, \Omega>1$.

Now we will quote some results of B. Szewc ([7]). For $\varepsilon \in\left[0, \min _{a \in A}(1-\xi(a)) / 2\right) \cup$ $\mathbb{N}$, let

$$
\varphi_{\varepsilon}(x)=\left[\sum_{n=0}^{\infty} \sum_{a \in \tilde{A}}\left|\left(f^{n}\right)^{\prime}(f(a))\right|^{-\eta(a)} \frac{1}{\left|x-f^{n+1}(a)\right|^{\varepsilon+\xi(a)}}\right]+1 .
$$

Let $\mathscr{J}$ denote the set of all components of $I \backslash B$. B. Szewc defines for $\rho: I \backslash B \rightarrow \mathbb{R}$

$$
\|\rho\|_{\varepsilon}=\sup _{J \in \mathscr{I}} \sup _{\boldsymbol{J}} \frac{|\rho|}{\varphi_{\varepsilon}} .
$$

If additionally $\rho$ is Lipschitz on compact subsets of $I \backslash B$, then Szewc also defines

and

$$
|\rho|_{(0)+1}=\sup _{J \in \mathscr{J}} \operatorname{essup} \frac{|\rho|}{\varphi_{1}}
$$

$$
\|\rho\|_{(0)+1}=\max \left\{\|\rho\|_{\varepsilon},|\rho|_{(0)+1}\right\} .
$$

He considers the space $C_{f, \varepsilon}^{(0)+1}$ of all functions $\rho$ which are Lipschitz on compact subsets of $I \backslash B$ and such that $\|\rho\|_{(0)+1}<+\infty$.

Let $\phi$ be the operator on $L^{1}(\lambda)$ given by the formula

$$
\phi(\psi)=f_{*}(\psi)-\left(\int \psi d \lambda\right) \cdot \phi \quad \text { for } \psi \in L^{1}(\lambda)
$$

(recall that $f_{*}$ is the Perron-Frobenius operator for the Lebesgue measure $\lambda$ ). 
B. Szewc proved that $C_{f, \varepsilon}^{(0)+1}$ is a Banach space ([7, remark 4.3(b)]) invariant with respect to $f_{*}$ and $\phi$, and there exist constants $\Gamma>0, \rho \in(0,1)$ such that for $\psi \in C_{f, \varepsilon}^{(0)+1}$

$$
\left\|\phi^{n}(\psi)\right\|_{L^{1}} \leq \Gamma \cdot \rho^{n}\|\psi\|_{(0)+1} .
$$

LeMMA 14. If $\Delta \in V_{0}^{M-1} f^{-i} A$, then $\phi^{M}\left(\varphi \cdot \chi_{\Delta}\right) \in C_{f, e}^{(0)+1}$ and $\left\|\phi^{M}\left(\varphi \cdot \chi_{\Delta}\right)\right\|_{(0)+1} \leq P$, where $P$ is a constant which does not depend on $\Delta$ nor on $M$.

Proof. The proof of this lemma follows easily from the considerations conducted in the proof of proposition 5.4 in [7].

LEMMA 15. There exists $\Gamma_{1}>0$ such that for all $M, n, l \in \mathbb{N}, l \geq M$,

$$
D\left(\bigvee_{0}^{M-1} f^{-i} \mathscr{A}, \bigvee_{M+1}^{M+l+n-1} f^{-i} \mathscr{A}\right) \leq \Gamma_{1}\left(\frac{N}{\rho}\right)^{M} \cdot \rho^{l}
$$

where $N$ denotes the number of elements of $\mathscr{A}$ and $\rho$ is the constant from (41).

Proof. Using an argument analogous to that in the proof of lemma 13 in [3] we show that

$$
D\left(\bigvee_{0}^{M-1} f^{-i} \mathscr{A}, \bigvee_{M+1}^{M+l+n-1} f^{-i} \mathscr{A}\right) \leq 2 \cdot \sum_{\Delta \in \bigvee_{0}^{M-1} f^{-i} \mathscr{A}}\left\|f_{*}^{l}\left(\varphi \cdot \chi_{\Delta}\right)-\mu(\Delta) \varphi\right\|_{L^{1}}
$$

Observe that $f_{*}^{\prime}\left(\varphi \cdot \chi_{\Delta}\right)-\mu(\Delta) \varphi=\phi^{\prime}\left(\varphi \cdot \chi_{\Delta}\right)$. In view of (41) and lemma 14 we have $\left\|\phi^{l}\left(\varphi \cdot \chi_{\Delta}\right)\right\|_{L^{1}} \leq \Gamma \cdot \rho^{l-M} P$.

LEMMA 16. If $l \geq 3 M$ then

$$
D\left(\bigvee_{0}^{M-1} f^{-i} \mathscr{A}, \bigvee_{M+l}^{M+l+n-1} f^{-i} \mathscr{A}\right) \leq \Gamma_{1}\left(\frac{N^{2}}{\rho^{3}}\right)^{M} \rho^{l}+M \frac{\Gamma_{2}}{(\sqrt[5]{\nu})^{M}}
$$

where $\Gamma_{2}>0$ does not depend on $M$ nor on $n$.

Proof. By theorems 2 and 3 the atoms of $V_{0}^{M-1} f^{-i} \mathscr{A}$ are contained in the atoms of $\mathscr{A}^{\prime}$, up to atoms whose sum has measure not greater than 2 card $\mathscr{A}^{\prime} R \sqrt[s]{d} /(\sqrt[s]{\nu})^{M}$. Hence we have

$$
\begin{aligned}
D( & \left.\bigvee_{0}^{M-1} f^{-i} \mathscr{A}^{\prime}, \bigvee_{M+l}^{M+l+n-1} f^{-i} \mathscr{A}\right) \\
& \leq D\left(\bigvee_{0}^{M-1} f^{-i}\left(\mathscr{A}^{\prime} \vee \bigvee_{0}^{M-1} f^{-j} \mathscr{A}\right), \bigvee_{M+l}^{M+l+n-1} f^{-i} \mathscr{A}\right) \\
& \leq D\left(\bigvee_{0}^{M-1} f^{-i}\left(\bigvee_{0}^{M-1} f^{-j} \mathscr{A}\right), \bigvee_{M+l}^{M+l+n-1} f^{-i} \mathscr{A}\right)+2 M 2 \operatorname{card} \mathscr{A}^{\prime} \frac{R \sqrt[s]{d}}{(\sqrt[s]{\nu})^{M}}
\end{aligned}
$$

but $\bigvee_{0}^{M-1} f^{-i}\left(\bigvee_{0}^{M-1} f^{-j} \mathscr{A}\right)=\bigvee_{0}^{2 M-2} f^{-i} \mathscr{A}$, and we can use lemma 15 to estimate $D\left(\bigvee_{0}^{2 M-2} f^{-i} \mathscr{A}, \bigvee_{M+l}^{M+l+n-1} f^{-i}(\mathscr{A})\right.$.

THEOREM 4. There exist constants $P>0, p \in(0,1)$ such that for arbitrary $n, k, l \in \mathbb{N}$ we have

$$
D\left(\bigvee_{0}^{n-1} f^{-i} \mathscr{A}, \bigvee_{n+1}^{n+1+k-1} f^{-i} \mathscr{A}\right) \leq P \cdot p^{l}
$$

Proof. We proceed in the same way as F. Hofbauer and G. Keller in the proof of $[3$, theorem 4$]$. 
Fix $\delta, 0<\delta<\frac{1}{4}$ such that $\rho\left(N^{2} / \rho^{3}\right)^{\delta}<1$. It suffices to prove the theorem for sufficiently large $l$ and $n \geq[\delta l]+1$.

Put $M:=[\delta l]+1, m:=n-M$. If $l$ is sufficiently large, then $M$ is so large that we can use all the lemmas proved above. In view of lemma 13(b)

$$
\begin{aligned}
D\left(\bigvee_{0}^{n-1} f^{-i} \mathscr{A}, \bigvee_{n+l}^{n+l+k-1} f^{-i} \mathscr{A}\right) & \leq D\left(\bigvee_{0}^{n-1} f^{-i} \mathscr{A}^{\prime}, \bigvee_{n+l}^{n+l+k-1} f^{-i} \mathscr{A}\right) \\
& \leq D\left(\mathscr{A}_{m, M}, \bigvee_{n+l}^{n+l+k-1} f^{-i} \mathscr{A}\right)+2 S / \Omega^{M}
\end{aligned}
$$

Put $\mathscr{G}:=\bigvee_{n+l}^{n+l+k-1} f^{-i} \mathscr{A}$. Performing estimations similar to those of the proof of theorem 4 of [3] and using lemma 13(a), (c) we prove that

$$
D\left(\mathscr{A}_{m, M}, \mathscr{G}\right) \leq\left(S / \Omega^{M}\right)+D\left(\bigvee_{0}^{M-1} f^{-i} \mathscr{A}^{\prime}, \underset{M+l}{M+l+k-1} f^{-i} \mathscr{A}\right)
$$

Notice, that $\delta<\frac{1}{4}$, so $3 M=3[\delta l]+1 \leq l$ and we can use lemma 16. We obtain

$$
D\left(\mathscr{A}_{m, M}, \mathscr{G}\right) \leq \frac{S}{\Omega^{M}}+\Gamma_{1}\left(\frac{N^{2}}{\rho^{3}}\right)^{M} \rho^{l}+M \frac{\Gamma_{2}}{(\sqrt[s]{\nu})^{M}} .
$$

But $M=[\delta l]+1,\left(N^{2} / \rho^{3}\right)^{\delta} \rho<1$, so the last inequality together with (42) gives an estimate for $n \geq[\delta l]+1$ and $n$ sufficiently large.

\section{Concluding remarks; proof of theorem 1}

In $\S 1$ we have formulated the main result of this paper, theorem 1 , which implies other important theorems like Log-log laws, integral tests, etc.... Now we are in the position to prove theorem 1:

Let $F$ and $\left(S_{t}\right)_{t \geq 0}$ be as in theorem 1. To prove theorem 1 (ii) it suffices to know that $F \circ f^{i}, i=0,1, \ldots$, can be treated as a functional of some process $\left(\zeta_{n}\right)_{n \in \mathbb{N}}$ and to check that the assumptions of theorem 7.1 of [6] are satisfied in this case.

Define, for $n \in \mathbb{N}$, a random variable $\xi_{n}$ on $\left.([0,1]), \mathscr{B}, \mu\right)$ by the formula

$$
\xi_{n}(x)=j \quad \text { if } f^{n}(x) \in\left[a_{j}, a_{j+1}\right),
$$

where $a_{i}, i=0, \ldots, s_{0}$ are the points of $A$.

In view of theorem 2 the process $\left(F \circ f^{i}\right)_{i=0,1, \ldots}$ can be treated as a functional of 'label-process' $\left(\zeta_{n}\right)_{n=0,1, \ldots}$. Now by using theorems 2,3 and 4 , we check as in the proof of theorem 4 in [3] that the assumptions of theorem 7.1 of [6] are satisfied.

In $\S 1$ we have assumed the continuity of $f$. This assumption is not necessary and was made only for simplicity. It suffices to assume the continuity of $f$ on components of the set $I \backslash A$.

\section{REFERENCES}

[1] R. Bowen. Bernoulli maps of an inverval. Israel J. Math. 28 (1977), 161-168.

[2] M. I. Gordin. The central limit theorem for stationary processes. Soviet Math. Dokl. 10 (1969), 1174-1176. 
[3] F. Hofbauer, \& G. Keller. Ergodic properties of invariant measures for piecewise monotonic transformations. Math. Zeit. 180 (1982), 119-140.

[4] G. Keller. Un theoreme de la limite central pour une class de transformations monotonnes par morceaux. C.R. Acad. Sc. Paris, Serie A, 291 (1980).

[5] M. Misiurewicz. Absolutely continuous measures for certain maps of an inverval. Publ. Math. IHES 53 (1981), 17-51.

[6] W. Phillipp \& W. Stout. Almost sure invariance principles for partial sums of weakly dependent random variables. Mem. Amer. Math. Soc. 161 (1975).

[7] B. Szewc. Perron-Frobenius operators in the spaces of smooth functions on an interval. Thesis.

[8] W. Szlenk. Some dynamical properties of certain differentiable mappings of an inverval. Bol. de la Soc. Mex. 24, No. 2, (1979), 57-82.

[9] S. Wong. A central limit theorem for piecewise monotonic mappings of the unit interval. Ann. Prob. (1979), 500-154. 